

\section{DISCLAIMER}

This report was prepared as an account of work sponsored by an agency of the United States Government. Neither the United States Government nor any agency thereof, nor any of their employees, make any warranty, express or implied, or assumes any legal liability or responsibility for the accuracy, completeness, or usefulness of any information, apparatus, product, or process disclosed, or represents that its use would not infringe privately owned rights. Reference herein to any specific commercial product, process, or service by trade name, trademark, manufacturer, or otherwise does not necessarily constitute or imply its endorsement, recommendation, or favoring by the United States Government or any agency thereof. The views and opinions of authors expressed herein do not necessarily state or reflect those of the United States Government or any agency thereof. 


\section{DISCLAIMER}

Portions of this document may be illegible electronic image products. Images are produced from the best available original document. 


\title{
A CORRELATION OF AIR-COUPLED ULTRASONIC AND THERMAL DIFFUSIVITY DATA FOR CFCC MATERIALS
}

\author{
T. A. K. Pillai ${ }^{*}$, W.A. Ellingson, and J.G. Sun
}

Energy Technology Division

Argonne National Laboratory

Argonne, IL 60439

T.E. Easler and A. Szweda

Advanced Ceramics Program

Dow Corning Corporation

Midland, MI 48686

\begin{abstract}
An air-coupled (non contact) through-transmission ultrasonic investigation has been conducted on 2D multiple ply Nicalon ${ }^{\mathrm{TM}} \mathrm{SiC}$ fiber/SiNC CFCC panels as a function of number of processing cycles. Corresponding thermal diffusivity imaging was also conducted. The results of the air-coupled ultrasonic investigation correlated with thermal property variations determined via infrared methods. Areas of delaminations were detected and effects of processing cycles were also detected.
\end{abstract}

\section{INTRODUCTION}

When applying evaluation and characterization methods to materials processing, care must be exercised to avoid any intrusion in to the manufacturing process. Air-coupled ultrasonic inspection offers such a choice for evaluating materials during their processing steps. Since air is part of the natural environment, this method offers

* Permanent Address: Department of Physics, University of Wisconsin - LaCrosse, LaCrosse, WI 54601 
the least damage during the processing cycles of materials. Traditional water-coupled (or other contact methods) offer no such non-destructive advantages for uncured composite materials during their processing. Currently, due to the improvement of technology in the making of air-coupled transducers, this method of evaluation is becoming popular. Efficient air-coupled transducers and associated instrumentation are now commercially available. A comprehensive description of the state of the art of such transducers and their NDE applications have been provided by Grandia, et al. [1].

Because of such advantages, we have been led to explore the applicability of aircoupled ultrasonic NDE method to CFCC materials during their processing cycles. We present here our preliminary results of such an investigation on multiple ply SYLRAMIC ${ }^{\mathbf{T M}}$ composites fabricated using a Polymer Impregnation and Pyrolysis (PIP) process. We have also performed, for comparison purposes, thermal diffusivity mapping of the same panels using infrared NDE methods. The results of the two NDE methods and their correlation are presented.

\section{SAMPLE DESCRIPTION}

SYLRAMICTM composites are fabricated using a Polymer Impregnation and Pyrolysis (PIP) process at Dow Corning Corporation. The SYLRAMICTM composites for this study are reinforced with Nicalon ${ }^{\mathrm{TM}} \mathrm{SiC}$ fibers, which are small-diameter, textile-grade ceramic fibers. As shown in the PIP process schematic in Figure 1,

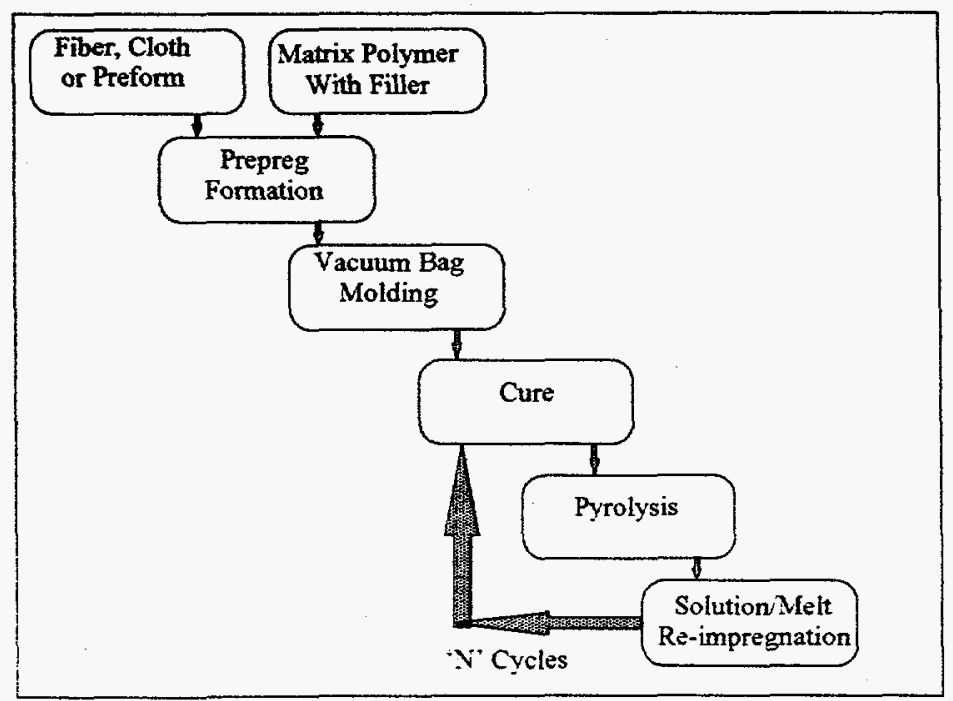

Figure 1: Schematic of the PIP Process 
the matrix-precursor polymer is first impregnated into the fiber architecture of choice, and then cured by conventional methods.

Both the initial shaping and fabrication of the composite are carried out using lowtemperature processing equipment. The composite is then pyrolyzed to temperatures greater than $1000^{\circ} \mathrm{C}$ to convert the pre-ceramic matrix polymer into a ceramic.

Subsequent impregnation and pyrolysis steps are carried out to achieve the desired final density or open porosity, as illustrated in Figure 2. The porosity values shown in Figure 2 are determined by a modification of the ASTM designation C 373-72 [2].

The composites used in this study are comprised specifically of ceramic-grade Nicalon ${ }^{\mathrm{TM}} 8 \mathrm{HS}$ fabric in a warp-aligned symmetric lay-up of 8 and 16 plies, with a proprietary interface coating applied to the fabric, and a silicon-carbon-nitrogen matrix chemistry derived from a preceramic polymer.

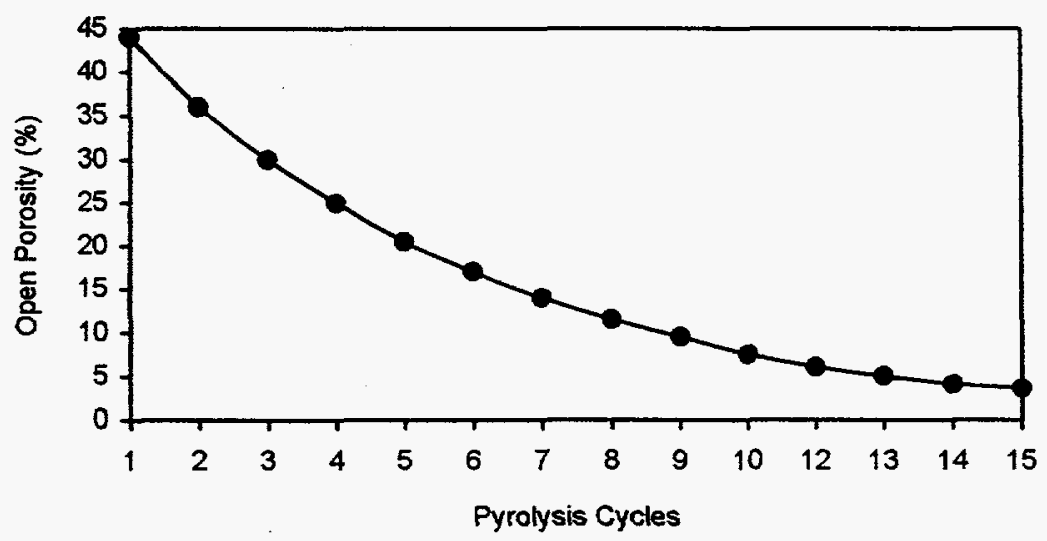

Figure 2: Open Porosity as a Function of PIP Cycle

The original $30.5 \mathrm{~cm} \mathrm{x} 30.5 \mathrm{~cm}$ panel was cut into progressively smaller pieces to facilitate the evaluation of potential sample-size effects on densification behavior during processing. Delaminations were introduced into some of the pieces to serve as "known" defects which could be observed as densification of the material progressed. 


\section{AIR-COUPLED ULTRASONIC IMAGING}

The samples were imaged by a traditional C-scan imaging system using a pair of transducers in a coaxial transmission geometry. A functional schematic diagram of the air-coupled ultrasonic imaging system is shown in Figure 3.

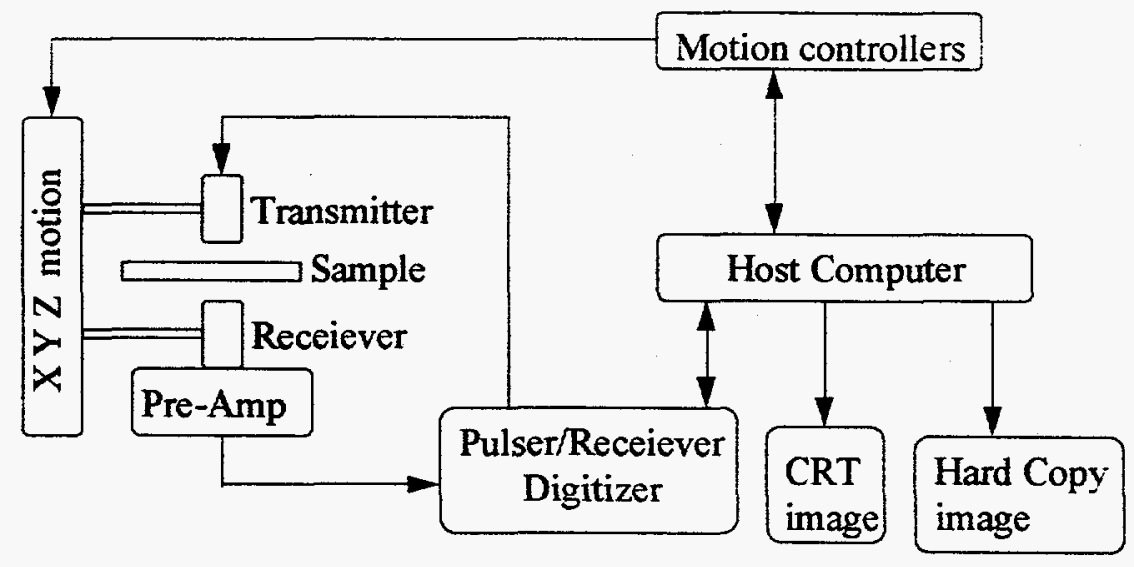

Figure 3: Functional Block Diagram of the Air-Coupled Ultrasonic System

The matched pair of air-coupled transducers operated at a central frequency of 400 $\mathrm{kHz}$, and their focal length was about $3.8 \mathrm{~cm}$. The transducers were aligned so that they were focused on to the sample. Using these transducers, a C-scan image was built up with a $0.08 \mathrm{~cm}$ step size. The X-Y motion and the data acquisition and processing was controlled by the host Pentium computer. Acoustic waves incident on to the sample traverse through the sample and the first transmitted signal received by the receiving transducer is pre-amplified by a low noise high gain pre-amp attached to the back of the receiver. These signals are further gated and amplified, and the average peak-in-the gate(PIG) is used to produce an image in a 16-color coded scale. The resulting image consisted of a large number of pixels whose colors depended on the transmitted amplitude. The color of the image thus depends on the physical property of the medium. As acoustic waves propagate through the medium they are scattered by defects such as pores or delaminations. The through transmitted amplitude of the waves through the defective areas of the sample will be quite 
different from the non-defective regions. Thus areas of non-uniform density, delaminations or porosity etc., are clearly distinguishable, as they will appear as different color-codes on the scanned image.

\section{THERMAL (INFRA RED) IMAGING}

The thermal imaging system uses the method of Parker et al. [3] to calculate thermal diffusivity. A diagram of the experimental set up is shown in Figure 4 . This method requires that a thermal pulse of short duration be incident on the front surface of a specimen and that the temperature of the back surface be monitored as a function of time. This is accomplished in the current study by heating the front surface with a 6 $\mathrm{kJ}$ photographic flash lamp (with a pulse duration of less than $8 \mathrm{~ms}$ ) and monitoring the back-surface temperature with a commercially available focal-plane-array infrared camera.

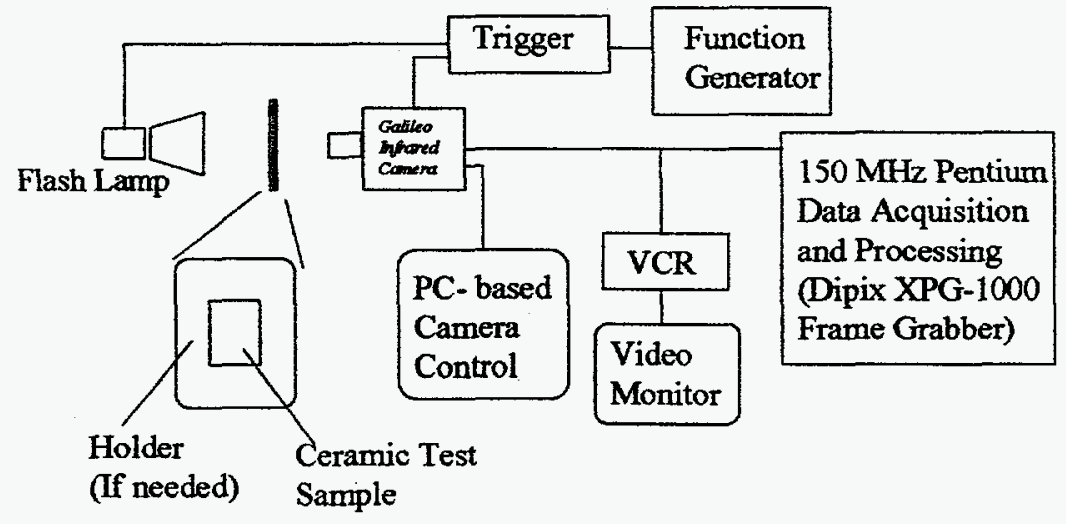

Figure 4: Experimental Setup used for Thermal Diffusivity Imaging

The thermal diffusivity was determined by the "half-rise time" method $[3,4,5]$. As an additional means of assessing data accuracy, the "quarter-rise time" and the "three-quarter-rise time" were also determined for each pixel, according to an extension of the Parker et al procedure suggested in ASTM specification E 1461-92, [6]. The resulting average thermal diffusivity values were assembled to form a 'diffusivity image' of the sample. 
The camera used in the present study was equipped with a $3.5 \mu \mathrm{m}$ optical band pass lens system and an indium antimonide (InSb)-based detector system. Images were acquired digitally by a pentium PC with an on-board frame grabber in real time at 256 $\times 256$ pixel 12-bit resolution. Image acquisition (to a speed of $1400 \mathrm{~Hz}$ ) and analysis were performed with locally written software. To minimize lateral heat flow, test specimens were insulated at the specimen edges.

\section{RESULTS AND DISCUSSION}

Air-coupled ultrasonic C-scan images of the 16 ply SYLRAMIC ${ }^{\text {TM }}$ panels after 5 PIP cycles are shown in Figure 5a. The corresponding thermal diffusivity images are shown in Figure $5 \mathrm{~b}$. Both set of images show overall uniformity. No delaminations or other defects are found in these samples. The correlation between the two images are very good. The smaller samples of the set $(3 \mathrm{~cm}$ or less in width) could not be successfully imaged using the air-coupled ultrasonic system due to the predominant edge scattering effects of acoustic waves. The uniformity of the 16 ply samples remained through the subsequent PIP cycles, hence their images are not reproduced here again after the 15 PIP cycles.

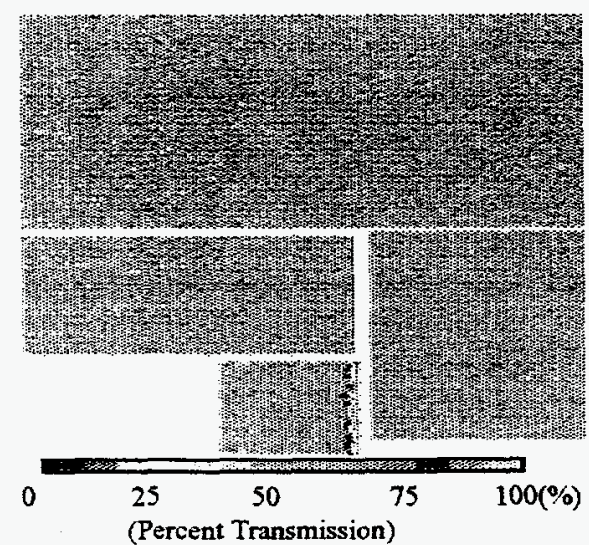

Figure 5a: Air-coupled Ultrasonic Image of thel6 ply panel after 5 PIP cycles

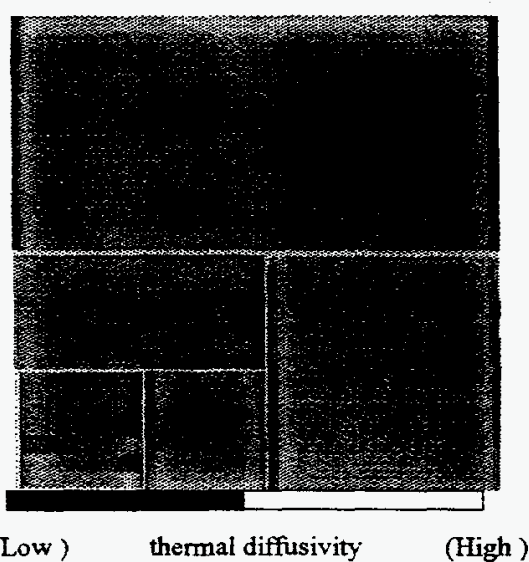

Figure 5b: Thermal diffusivity Image of the 16 ply panel after 5 PIP eycles 
The images of the 8 ply SYLRAMICTM panels after 5 PIP cycles are shown in Figures $6 \mathrm{a}$ and $6 \mathrm{~b}$. In contrast to the images of the 16 ply samples, these samples show areas of delaminations clearly. It must be reported here that the air-coupled ultrasonic scan was performed without prior knowledge of the condition of the panel. It is seen from Figures $6 \mathrm{a}$ and $6 \mathrm{~b}$ that the ultrasonic images match very well with the thermal diffusivity images. One noticeable difference is that the boundary of the delaminated areas are much sharper in the ultrasonic images. Since the air-coupled ultrasonic C-scan system is currently operated in the through-transmission mode, no quantitative evaluations are made regarding the depth of these delaminations in the panels. Other modes of operation such as the pitch-catch method may be used to extract depth information.

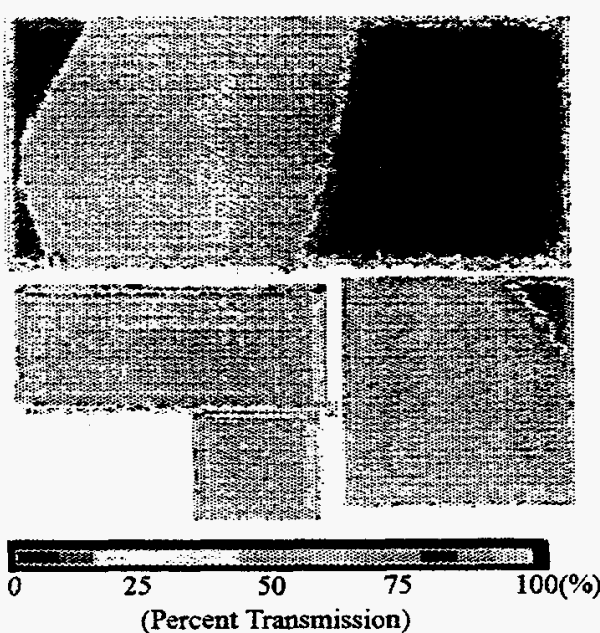

Figure 6a: Air-coupled Ultrasonic Image of the 8 ply panel after 5 PIP cycles

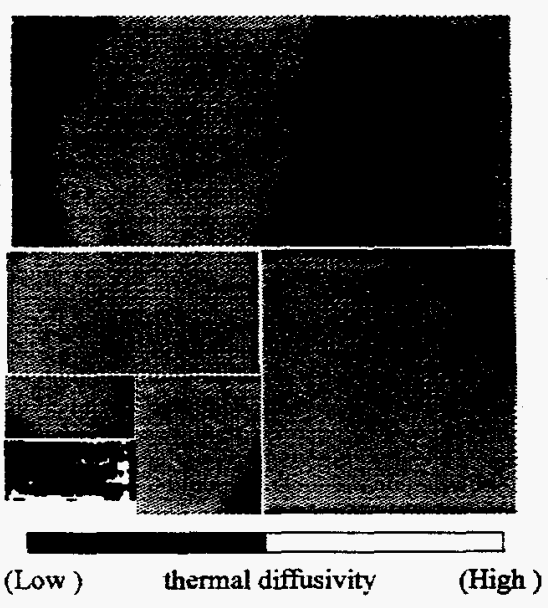

Figure 6b: Thermal diffusivity Image of the 8 ply panel after 5 PIP cycles

It is evident from the results reported here that air-coupled ultrasonic method has considerable potential as a truly non-invasive NDE tool in cases where the test material may be sensitive to traditional acoustic coupling agents. This is especially true in the types of materials used in the present study. Air-coupled NDE provides a suitable method for evaluating materials during their processing cycles. The present study also show that the results of air-coupled investigation correlate well with thermal property evaluations. 


\section{ACKNOWLEDGMENTS}

This Work was funded jointly by Dow Coming and the U.S. Department of Energy, Office of Industrial Technologies, Office of Energy Efficiency and Renewable Energy, cooperative agreement DE-FC02-92CE40993. Such support does not constitute an endorsement by DOE of the views expressed in this work.

\section{REFERENCES}

1. W. A. Grandia and C. M. Fortunko, "NDE Applications of Air-Coupled Ultrasonic Transducers", IEEE Ultrasonics, Ferroelectrics, and Frequency Control, IEEE International Symposium, Proceedings, Vol 1, pp: 697 -709, (1995)

2. American Society for Testing and Materials, "Standard Test Method for Water Absorption, Bulk Density, Apparent Porosity, and Apparent Specific Gravity of Fired Whiteware Products", Designation C 373-72, ASTM, Philadelphia, (1982).

3. W. J. Parker, R. J. Jenkins, C. P. Butler and G. L. Abbott, "Flash method of determining thermal diffusivity, heat capacity, and thermal conductivity", J. Appl. Phys., 32(9), 1679-1684, (1961).

4. W. A. Ellingson, S.A. Rothermel, J.F. Simpson, "Nondestructive Characterization of Ceramic composites Used as Combustor Liners in Advanced Gas Turbines", J.of Eng. For Gas Turbines and Power, Transactions of ASME, Vol. 118, pp 486-490, 1996.

5. S. Ahuja, W.A. Ellingson, J.S. Steckenrider and S. King, "Thermal Diffusivity Imaging of Continuous Fiber Ceramic Composite Materials and Components", Proc. of 23rd International Thermal Cond. Conf. Eds: K.E. Wilkes, R.B. Dinwiddie and R.S. Graves, pp $311-321,1996$.

6. American Society for Testing and Materials, "Standard Test Method for thermal diffusivity of solids by the flash method", Annual book of ASTM Standards, Vol. 14.02 (General Test Methods, Nonmetal; Laboratory Apparatus; Statistical Methods; Forensic Sciences), 883-890, ASTM, Philadelphia, (1994). 